\title{
Anxiety and mortality risk in community-dwelling elderly people
}

Isabelle Carrière, Joanne Ryan, Joanna Norton, Jacqueline Scali, Robert Stewart, Karen Ritchie and Marie Laure Ancelin

\section{Background}

There are conflicting data on the role of anxiety in predicting mortality.

\section{Aims}

To evaluate the 10-year mortality risk associated with anxiety in community-dwelling elderly people.

\section{Method}

Using data from 718 men and 1046 women aged 65 years and over, gender-stratified associations of anxiety symptoms (Spielberger State-Trait Anxiety Inventory, third tertile) and current DSM-IV anxiety disorder including generalised anxiety disorder (GAD) and phobia with all-cause and cardiovascular mortality were determined.

\section{Results}

In women, mortality risk was increased for anxiety disorder and GAD in multivariate Cox models (hazard ratio $(H R)=1.53$, 95\% Cl 1.02-2.27 and $\mathrm{HR}=2.04,95 \% \mathrm{Cl} 1.08-3.86$ respectively), whereas for phobia it was nearly significant $(\mathrm{HR}=1.52,95 \% \mathrm{Cl}$ 0.94-2.47). Anxiety trait symptoms became non-significant as a result of the confounding effect of depressive symptoms. Anxiety disorder was associated with cardiovascular mortality in univariate analysis $(\mathrm{HR}=2.42$, $95 \% \mathrm{Cl} 1.16-5.07)$. No significant associations were found in men.

\section{Conclusions}

Our study suggests a gender-specific association of anxiety and mortality.

\section{Declaration of interest}

None.
In community populations, the association between depression or depressive symptoms and mortality is now well established, particularly in elderly people. ${ }^{1,2}$ In contrast there are conflicting data on associations with anxiety. A meta-analytic review of seven studies of the impact of overall neurosis on mortality in elderly people ${ }^{3}$ concluded that although there was a tendency for anxiety to be associated with mortality, the combined effect failed to reach significance $(P=0.08)$. More recent studies (online Table DS1) have confirmed the results of this meta-analysis in finding no significant association between anxiety and mortality risk, ${ }^{4,5}$ whereas others have found anxiety to be a risk factor for mortality $^{6-12}$ and some even to be a protective factor. ${ }^{13,14}$ These conflicting data may be because of (a) the heterogeneity in study design (clinical sample $v$. general population) and in sample size, gender and age with age-related differences both in the type of anxiety $^{15}$ and causes of mortality, (b) the severity of anxiety (clinical diagnosis $v$. subclinical symptoms and presence of comorbidity), (c) the different anxiety disorder subtypes (for example panic disorder, phobia or generalised anxiety disorder $(\mathrm{GAD})$ ), and (d) anxiety evaluation (diagnosis $v$. self-report scales). Several methodological issues may also contribute to observed inconsistencies; (a) the lack of specific anxiety symptom scales that may also screen for depressive symptoms, (b) the lack of a control for comorbid depression and other confounders, and (c) the statistical approach used to take into account age, notably in elderly populations where both the mortality risk and the major confounders are strongly related to age. All previously cited studies ${ }^{4-6,8,9,11,12,14}$ except one ${ }^{10}$ used time-on-study (i.e. time since inclusion data) instead of age as the timescale in Cox's regression models taking age as a covariate, although this method can induce bias on effect estimates. ${ }^{16}$

Large epidemiological studies in this area using validated caseidentification procedures taking into account both anxiety subtypes and psychiatric comorbidity as well as appropriate analyses are thus still lacking. The purpose of this study was to evaluate the 10-year mortality risk of anxiety measured both with a standardised psychiatric interview examining different anxiety subtypes and a validated self-report symptom scale, in a large cohort of community-dwelling elderly people.

\section{Method}

\section{Study sample}

The design of the ESPRIT study and participant recruitment have been published in detail elsewhere. ${ }^{17}$ Participants were recruited over a 2-year period from 1999 to 2001, by random selection from the electoral rolls in Montpellier, France. Eligible individuals, who were at least 65 years of age and not living in institutions, were invited to participate and provided written informed consent. Participants were administered a number of standardised questionnaires by trained staff and underwent clinical examinations at baseline, 2 years, 4 years, 7 years and 10 years in a dedicated centre or at home. Ethics approval for the ESPRIT study was granted by the ethics committee of the University Hospital of Kremlin-Bicêtre (France).

Vital status was available for all 2189 dementia-free participants included in the study, however, 425 participants had missing data for at least one baseline adjustment variable or anxiety. The present analysis was thus conducted on 1764 participants (718 men and 1046 women). Compared with the analysed sample, those not included in this analysis were more likely to be older $(P=0.0001)$ and obese $(P=0.003)$, have a lower level of education $(P=0.006)$, a lower level of income $(P<0.0001)$, low high-density lipoprotein $(\mathrm{HDL})$ cholesterol $(P=0.002)$, have activity limitations $(P<0.0001)$, low physical activity $(P=0.0009)$ and cognitive impairment $(P=0.007)$, but they were less likely to have anxiety disorders $(P=0.006)$ and trait anxiety symptoms $(P=0.04)$. There were no significant differences regarding chronic disease and depression. 


\section{Mortality end-point}

At each follow-up, participants were contacted and when they could not be reached, vital status was ascertained by contacting relatives and general practitioners or the civil registry. The vital status was obtained for all participants. In the case of death we obtained hospital records including the exact date of death and information from general practitioners. An independent committee reviewed records to determine immediate and underlying causes of death, according to ICD-10 codes. ${ }^{18}$ The closing date for the present analysis was the end of 2011. All-cause mortality was the principal outcome and cause-specific mortality was also examined. Immediate or underlying cause coded between C00 and C97 or between D00 and D48 defined cancer mortality and between I00 and I78 or equal to R960 cardiovascular mortality.

\section{Anxiety disorders and symptoms}

A standardised psychiatric examination, the Mini-International Neuropsychiatric Interview (MINI), was used at baseline to investigate lifetime psychiatric disorder. ${ }^{19}$ This interview has previously been validated in France. ${ }^{20}$ The interview was administered by trained nurses and psychologists. The MINI provides an extensive examination according to DSM-IV criteria, ${ }^{21}$ with diagnostic algorithms applied to assess 'caseness'. With regard to anxiety disorders, it provides diagnoses of both current and past GAD, social phobia, agoraphobia, obsessive-compulsive disorder (OCD), panic and post-traumatic stress disorder (PTSD). Cases detected by the MINI were reviewed by a panel of psychiatrists to validate the initial diagnosis. ${ }^{17}$ Spielberger's State-Trait Anxiety Inventory (STAI) was used to measure trait and state anxiety symptoms. ${ }^{22}$ The two scales are composed of 20 items, each rated on a four-point intensity scale. In the absence of a validated cut-off score in the elderly population, STAI trait and state scores were divided into tertiles, with the highest tertile being compared with the lowest two.

\section{Depressive symptomatology}

The MINI was also used for the diagnosis of current and past major depressive episodes, according to DSM-IV criteria. In addition, severity of depressive symptoms was assessed using the 20-item Center for Epidemiologic Studies - Depression scale (CES-D). ${ }^{23}$ For this analysis, participants were classified into one of three groups. ${ }^{24}$ 'Severe depressive symptomatology' included participants with a current major depressive episode or a CES-D score of 23 or over. 'Mild depressive symptomatology' was defined as a CES-D score between 16 and 22, and 'No depressive symptomatology' included participants with a CES-D score lower than 16.

\section{Sociodemographic and clinical variables}

The standardised interview included questions on demographic characteristics, level of education ( $\leqslant 5 v .>5$ years of education), mode of living (alone or not) and income as well as an inventory of all drugs used over the preceding month (including anxiolytic and antidepressant drugs) validated by presentation of the prescription or medication. Information was obtained on the type and quantity of alcohol daily consumption (teetotaller, 1-36 v. $>36 \mathrm{~g} /$ day) and tobacco use (classified as past, present or never users). Detailed medical questionnaires included history of cardiovascular diseases (angina pectoris, myocardial infarction, arteritis, cardiac insufficiency, arrhythmia, cardiovascular surgery and stroke), history of cancer with hospital admission during the past 2 years, respiratory disease (asthma attacks during the past year and chronic bronchitis), dyspnoea, hypertension ( $>160 / 95 \mathrm{mmHg}$ or treated) and diabetes (fasting glycaemia $>7 \mathrm{mmol} / \mathrm{l}$ or treated). Blood pressure was measured during the interview using a digital electronic tensiometer. A body mass index (BMI) between 25 and $30 \mathrm{~kg} / \mathrm{m}^{2}$ was classified as overweight, and a BMI greater than $30 \mathrm{~kg} / \mathrm{m}^{2}$ as obese. Cognitive impairment was defined as a Mini-Mental State Examination $(\mathrm{MMSE})^{25}$ score lower than 24 . The number of chronic diseases was calculated separately for cardiovascular diseases and for other disorders such as respiratory diseases, hypertension, dyspnoea, diabetes and cancer. Lipid levels including HDL cholesterol were evaluated in serum as described previously. ${ }^{26}$

With regard to activity limitations, mobility was assessed using the Rosow \& Breslau scale, ${ }^{27}$ which assesses ability to perform physical tasks requiring mobility and strength. The Lawton-Brody scale examining ability to carry out eight instrumental activities of daily living (five for men) was also used. ${ }^{28}$ Physical activity was assessed through three questions concerning gardening, walking and sport and categorised as low (never or less than $1 \mathrm{~h}$ per day), moderate (regularly or $1-2 \mathrm{~h}$ per day) and high (often or more than $2 \mathrm{~h}$ per day).

\section{Statistical analyses}

The chi-squared test was used to compare baseline characteristics of men and women. The Kaplan-Meier method was used to derive survival curves. The censoring date was the date of last follow-up or telephone contact for the 10-year follow-up. Cox regression analyses with delayed entry taking age as the basic timescale and birth as the time origin were used to investigate the impact of baseline anxiety disorder or anxiety symptoms on mortality. This method enables a better adjustment for age and is preferred, especially for an elderly sample, over the standard model that uses study time as the timescale when some covariates are strongly associated with age (for example chronic diseases, impairments). ${ }^{16}$ To investigate the role of each potential confounder, covariates that were associated with mortality at a level of $P<0.15$ (online Table DS2) in either men or women were first entered separately in the model. A multivariate model was then constructed with all possible confounders. A priori potential interactions with anxiety disorder or anxiety symptoms tested were gender, anxiolytic medication, depression symptoms and a history of cardiovascular diseases. As the interaction between gender and anxiety disorder approached significance $(P=0.07)$ all subsequent analyses were stratified by gender. All other interactions were non-significant $(P>0.20)$. The statistical analyses were performed using SAS, version 9.2 for Windows.

\section{Results}

Table 1 provides a gender comparison of the population in terms of the anxiety criteria (see online Table DS3 for a more detailed version including all potential confounders). Women were more likely to have current and past anxiety disorder, and anxiety trait or state symptoms. The two main subtypes of anxiety disorder (GAD and phobia) were also more frequent in women, whereas very few cases were observed of $\operatorname{OCD}(n=11)$, panic disorder $(n=6)$ and PTSD $(n=5)$. Among people with anxiety disorder, $55.2 \%$ and $54.8 \%$ were in the upper tertile of trait and state anxiety symptoms respectively.

The median (interquartile range, IQR) follow-up time for this analysis was $9.0(8.8-9.4)$ years. Of the 1046 women and 718 men, $150(14.3 \%)$ and $171(23.8 \%)$ died during the follow-up respectively. The mortality ascertainment was complete for all participants. The time of follow-up for censored participants 
Table 1 Percentage meeting anxiety criteria stratified by gender $(n=1764)^{\mathrm{a}}$

\begin{tabular}{|c|c|c|c|}
\hline \multirow[b]{2}{*}{ Anxiety criteria } & \multicolumn{2}{|c|}{$\%$} & \multirow[b]{2}{*}{$\chi^{2}, P$} \\
\hline & $\begin{array}{c}\text { Men } \\
(n=718)^{b}\end{array}$ & $\begin{array}{l}\text { Women } \\
(n=1046)^{\mathrm{b}}\end{array}$ & \\
\hline Current anxiety disorder $^{\mathrm{C}}$ & 9.3 & 19.9 & $<0.0001$ \\
\hline History of anxiety disorder & 18.0 & 32.2 & $<0.0001$ \\
\hline Generalised anxiety disorder only & 1.7 & 4.1 & 0.006 \\
\hline Phobia only & 5.8 & 12.9 & $<0.0001$ \\
\hline Spielberger Trait (top tertile) & 21.7 & 39.7 & $<0.0001$ \\
\hline Spielberger State (top tertile) & 24.5 & 35.8 & $<0.0001$ \\
\hline Anxiolytic medication & 6.7 & 13.2 & $<0.0001$ \\
\hline \multicolumn{4}{|c|}{$\begin{array}{l}\text { a. See online Table DS3 for a comparison for all baseline characteristics. } \\
\text { b. } n \text { differs for the following: current anxiety disorder } n=1708 \text {; history of anxiety } \\
\text { disorder } n=1715 \text {; generalised anxiety disorder only } n=1717 \text { and phobia only } n=1710 \text {. } \\
\text { c. Current anxiety disorder: current generalised anxiety disorder, social phobia, } \\
\text { agoraphobia, obsessive-compulsive disorder, panic disorder and post-traumatic } \\
\text { stress disorder. }\end{array}$} \\
\hline
\end{tabular}

ranged between 7.7 and 11.5 years (median 9.0, IQR = 8.9-9.5) and was not statistically different according to baseline anxiety disorder (Wilcoxon rank sum test, $P=0.35$ ). The crude comparison of the Kaplan-Meier survival curves according to gender and the presence of anxiety disorder at baseline (Fig. 1) shows a more favourable survival probability in non-anxious women (Log-Rank, $P<0.0001)$. In univariate Cox models adjusting for age and stratified by gender (Table 2), anxiety disorder was strongly associated with mortality in women only (hazard ratio $(\mathrm{HR})=1.76,95 \%$ CI 1.21-2.57). There was a similar association with anxiety trait symptoms that was of borderline significance $(\mathrm{HR}=1.38,95 \%$ CI $1.00-1.92)$. For anxiety state symptoms a non-significant positive association was found in both men and women ( $P=0.13$ and $P=0.10$ respectively). A history of anxiety disorder was not significantly associated with mortality in either men $(P=0.42)$ or women $(P=0.62)$.

When potential confounders (cardiovascular diseases, nonvascular chronic diseases, cognitive impairment, daily activity limitations, obesity and physical activity, hospital admission in the past 2 years, and depressive symptomatology) were entered separately in the Cox model (online Table DS4), the association between anxiety disorder and mortality in women remained virtually unchanged. In contrast, the association with trait symptoms in women was clearly attenuated after adjustment for depressive symptoms, becoming non-significant. The study of concordance between depressive symptomatology (CES-D $\geqslant 16$ or major depressive episodes) and anxiety in our sample indicated a high overlap between anxiety trait symptoms and depressive symptomatology, with a Kappa coefficient of 0.51 ; for anxiety state symptoms the Kappa was 0.36 and for anxiety disorders as low as 0.18 .

In the final multivariate model (Table 2) with the inclusion of all of the potential confounding factors, as well as education level, the relationship between anxiety disorder and mortality persisted in women $(\mathrm{HR}=1.53,95 \% \mathrm{CI} 1.02-2.27)$. When anxiolytic medication was added to this model the association also remained unchanged $(\mathrm{HR}=1.51,95 \% \mathrm{CI} 1.02-2.26, P=0.04)$, and no interaction was found between anxiety disorder and anxiolytic medication $(P=0.64)$.

In women with respect to the cause of mortality, anxiety disorder was not significantly associated with cancer mortality (42 deaths). There was a significant association between anxiety disorder and cardiovascular mortality (32 deaths) in univariate analysis $(\mathrm{HR}=2.42,95 \%$ CI $1.16-5.07, \quad P=0.02)$, but this association became non-significant after multivariate adjustment $(\mathrm{HR}=1.66,95 \%$ CI $0.76-3.62, P=0.20)$.

Considering subtypes of anxiety diagnoses, women with GAD or phobia only were found to be at an increased risk of all-cause mortality in univariate analyses (Table 3). Adjusting for depression slightly weakened the associations $(\mathrm{HR}=2.01,95 \%$ CI $1.07-3.76, P=0.03$ and $\mathrm{HR}=1.69,95 \%$ CI $1.06-2.70$, $P=0.03$ for GAD and phobia respectively). However, in spite of the relatively low numbers of deaths by diagnostic group, the relationship with GAD remained significant after multiple adjustments (HR $=2.0495 \%$ CI 1.08-3.86) and just failed to reach significance for phobia ( $\mathrm{HR}=1.52$ 95\% CI 0.94-2.47).

No significant associations were found in men irrespective of the anxiety disorder and symptoms in univariate and multivariate models.

\section{Discussion}

Our study in an elderly population shows that the relationship between anxiety and mortality appears to be gender specific. In women our findings suggest that anxiety disorders meeting strict diagnostic criteria are associated with a 53\% increased risk of death during the 10-year follow-up. This association remained significant when adjusting for a range of possible confounders at baseline including current depressive symptoms, activity limitations, vascular and other chronic diseases, suggesting that anxiety disorder is an independent risk factor for mortality in women, and probably for cardiovascular mortality. When considering the two main subtypes of anxiety disorder in elderly people, both GAD and to a lesser extent phobia, increased the risk of all-cause death in women. We also found an association between Spielberger trait symptoms and mortality in women, but this association was confounded by depressive symptoms. In contrast, no significant associations were found in men, either for anxiety disorder or anxiety symptoms.

\section{DSM-IV anxiety disorders and mortality}

Inconsistent results have been reported concerning anxiety disorder and mortality in elderly people. In the AMSTEL cohort of elderly people recruited through general practices and with a high level of dependency ( $26 \%$ with instrumental activities of daily living (IADL) dependency), no association was found between 10-year mortality and anxiety diagnosis. ${ }^{4}$ Two other studies, mainly in non-elderly adults found either a significant increased risk of death ${ }^{10}$ or no significant association. ${ }^{5}$ None of these studies examined gender-specific effects. The only prior study investigating a gender-anxiety interaction, the LASA cohort (see Table DS1), showed that anxiety disorder was associated with 7 -year mortality risk in men only. ${ }^{8}$ This contrasts with our findings of an increased mortality risk specific for women. Comparing our study with theirs, the overall prevalence of anxiety disorder was similar, but they found a much higher frequency of $\operatorname{GAD}(11.7 \%$ v. $3.1 \%)$ and a lower level of phobia (5.5\% v. $10 \%)$. However, the discrepancy with our results remains difficult to explain and further studies exploring gender interactions are needed.

\section{State and trait anxiety symptoms and mortality}

Few studies have either explored the association between anxiety symptomatology and mortality or differentiated state and trait anxiety in an elderly community-dwelling population (see Table DS1). Two studies reported an increased risk of all-cause mortality in participants aged 75 years and over ${ }^{11}$ and in adult men and 


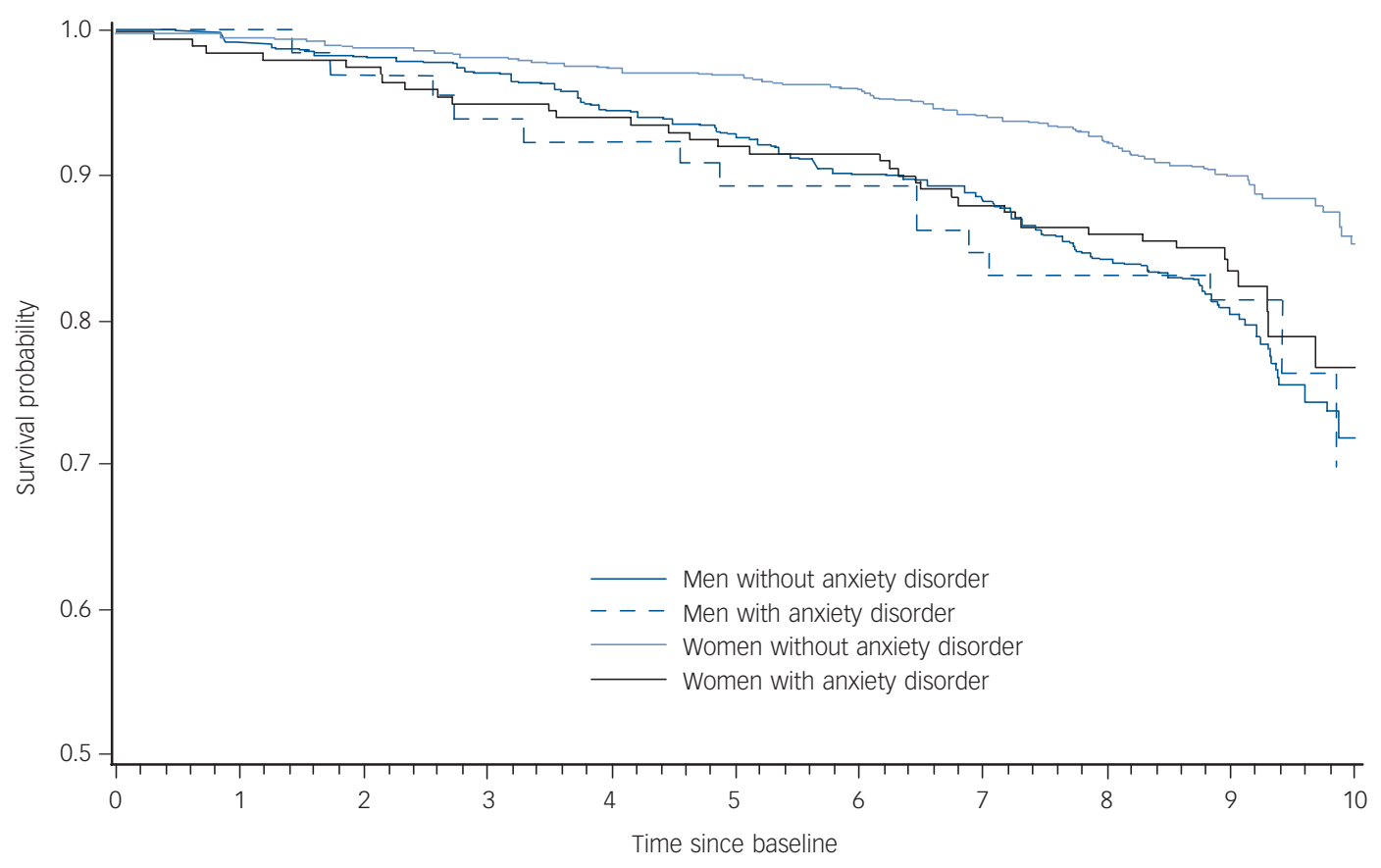

Fig. 1 Kaplan-Meier survival curves by gender and baseline anxiety disorder.

women. ${ }^{6}$ However, neither of these studies took into account depression as a possible confounder. An increased risk of mortality was reported in adult women using the three-item anxiety subscale of the Edinburgh Postnatal Depression Scale even after adjusting for depression. ${ }^{9}$ In the very large adult and elderly sample of the HUNT cohort, interaction by gender was not found but the association between anxiety symptoms ${ }^{29}$ and 3- to 6-year mortality was U-shaped with an increased risk for both the lowest and the highest quartiles of the distribution. In contrast with diagnostic tools however, the anxiety symptom scales generally examine the number and frequency of symptoms but they do not take into account their intensity. This may partially explained the poor overlap observed in our study between anxiety symptomatology and anxiety diagnostic. In women, a slight association between mortality and Spielberger trait symptoms disappeared after multiple adjustment. We found no evidence of a non-linear relationship (including U-shaped) (test of nonlinearity, $P \geqslant 20$ for Spielberger trait and state symptoms in men and women). We also found that Spielberger trait and state scales were highly linked to depressive symptomatology, which was the main confounder for the association with mortality. Hence, the difficulties encountered in assessing the specific effect of anxiety on mortality (different effect according to age, gender and subtype of anxiety) are amplified when dimensional symptomatology rather than diagnostic dichotomy is used for case identification because of the heterogeneity of the scale used and its correlation with depressive symptomatology.

\section{Anxiety and cardiovascular death}

In our cohort, unadjusted anxiety disorder was predictive of cardiovascular mortality in women, however, the association became non-significant after multiple adjustment probably as a result of lack of power. Cardiovascular disease (present in $24 \%$ of participants at baseline) did not appear to modulate the association with mortality. Only one study in an older elderly population (aged 75 years and over) has examined the association between high anxiety symptomatology and cause of death, finding a significantly increased risk of cardiovascular mortality, but not adjusting for depression. ${ }^{11}$ In a community-dwelling cohort of

\begin{tabular}{|c|c|c|c|c|c|c|}
\hline & \multicolumn{3}{|c|}{ Men } & \multicolumn{3}{|c|}{ Women } \\
\hline & Events/total, $n$ & $\mathrm{HR}(95 \% \mathrm{Cl})$ & $P$ & Events/total, $n$ & $\mathrm{HR}(95 \% \mathrm{Cl})$ & $P$ \\
\hline \multicolumn{7}{|l|}{ Univariate model } \\
\hline Anxiety disorder & $162 / 702$ & $1.02(0.58-1.78)$ & 0.96 & $136 / 1006$ & $1.76(1.21-2.57)$ & 0.003 \\
\hline Trait anxiety & $168 / 710$ & $1.04(0.72-1.50)$ & 0.85 & $147 / 1031$ & $1.38(1.00-1.92)$ & 0.05 \\
\hline State anxiety & $161 / 687$ & $1.30(0.93-1.83)$ & 0.13 & $137 / 973$ & $1.33(0.95-1.87)$ & 0.10 \\
\hline \multicolumn{7}{|l|}{ Multivariate model ${ }^{b}$} \\
\hline Anxiety disorder & & $1.00(0.57-1.78)$ & 0.99 & & $1.53(1.02-2.27)$ & 0.04 \\
\hline Trait anxiety & & $0.91(0.62-1.35)$ & 0.65 & & $1.22(0.83-1.78)$ & 0.32 \\
\hline State anxiety & & $1.18(0.82-1.70)$ & 0.39 & & $1.08(0.74-1.58)$ & 0.68 \\
\hline \multicolumn{7}{|c|}{$\begin{array}{l}\text { HR, hazard ratio. } \\
\text { a. See online Table DS4, for a more detailed version of this table with adjustments entered separately. Anxiety disorder: current generalised anxiety disorder, social phobia, } \\
\text { agoraphobia, obsessive-compulsive disorder and panic and post-traumatic disorder. Speilberger trait: top tertile, Speilberger state: top tertile. } \\
\text { b. Adjusted for number of cardiovascular diseases, number of non-vascular chronic diseases, cognitive impairment (Mini-Mental State Examination <24), mobility, instrumental } \\
\text { activities of daily living, obesity, physical activity, hospital admissions in the past } 2 \text { years, depressive symptomatology and education, and with age as time-scale. }\end{array}$} \\
\hline
\end{tabular}




\begin{tabular}{|c|c|c|c|c|c|c|}
\hline & \multirow[b]{2}{*}{ Censored, $n(\%)$} & \multirow[b]{2}{*}{ Deaths, $n(\%)$} & \multicolumn{2}{|c|}{ Univariate model } & \multicolumn{2}{|c|}{ Multivariate model $^{\mathrm{b}}$} \\
\hline & & & Hazard ratio $(95 \% \mathrm{Cl})$ & $P$ & Hazard ratio $(95 \% \mathrm{Cl})$ & $P$ \\
\hline No anxiety disorder & $708(83.79)$ & $98(74.24)$ & - & - & - & - \\
\hline Generalised anxiety disorder only & $29(3.43)$ & $12(9.09)$ & $2.37(1.30-4.33)$ & 0.005 & $2.04(1.08-3.86)$ & 0.03 \\
\hline Phobia only & $108(12.78)$ & $22(16.67)$ & $1.76(1.10-2.80)$ & 0.02 & $1.52(0.94-2.47)$ & 0.09 \\
\hline
\end{tabular}

adult women, anxiety symptomatology was also associated with a more than twofold increased risk of cardiovascular mortality. ${ }^{9}$

Studies of clinical populations with cardiac patients have reported conflicting results. A meta-analysis ${ }^{30}$ combining 12 studies published up to 2009 evaluating the effect of postmyocardial infarction found anxiety symptoms to be associated with a $36 \%$ and $47 \%$ increased unadjusted risk of adverse cardiac outcomes and all-cause mortality respectively. However, only three studies (two reporting association with cardiac mortality or recurrent myocardial infarction and one with cardiac mortality) remained significant in multivariate analysis. A more recent German study ( $74 \%$ males) reported an association between anxiety symptoms and a lower mortality rate in patients with coronary heart disease without a history of myocardial infarction, but in patients without post-myocardial infarction no effect of anxiety symptomatology was observed, whereas in the subgroup of patients with post-myocardial infarction and a poorer prognosis (with reduced ejection fraction) anxiety symptoms were associated with increased mortality. ${ }^{31}$ The severity of underlying cardiac disease might thus account for reported inconsistent results. Phobic symptoms were also found to be associated with higher cardiac mortality in women only, but the analysis did not adjust for depression. ${ }^{32}$ A higher vulnerability of elderly women with anxiety to cardiovascular mortality than men is possible and still has to be confirmed. If confirmed, then psychological interventions, as part of cardiac rehabilitation offered to individuals after cardiac events, should include anxiety detection and treatment.

\section{Gender-specific effects}

The discrepancy between published studies and the possible gender differences may be partly a result of the diagnostic tools used, which may detect different patterns of anxiety disorder. This point is of special importance in elderly populations where anxiety may be experienced differently and often takes a somatic form. ${ }^{33,34}$ In our sample, particularly among women, the frequency of phobia was relatively high, possibly because it is extensively explored by the MINI without application of organic disease exclusion criteria. We adjusted for a range of physical diseases but a residual effect of physical diseases on mortality may subsist. Cultural or generation differences relating to the social role of women in the family could also interact with the level of anxiety induced by stressful events such as loneliness, disease and dependency. The trajectory to death is also probably gender specific. It was shown that in the last year of life, women more often present with a progressive disability, ${ }^{35}$ whereas men are generally affected by rapid decline. Hormonal difference could also be involved. We have recently reported an association between oestrogen receptor polymorphisms and the risk of anxiety disorder in women, specifically, as well as the modifying effects of some oestrogen receptor polymorphisms on the association between hormonal treatment use and anxiety disorder as well as mortality in women. ${ }^{36,37}$

\section{Biological hypotheses}

There are a number of biological mechanisms that may be hypothesised to underlie the associations reported here, involving the hypothalamic-pituitary-adrenal (HPA) axis. In response to stressful conditions, chronic overactivity and dysregulation of the stress system can play a pivotal role in a number of critical biological processes, such as growth, intermediary metabolism and diabetes, immune and inflammatory reactions as well as vascular and central nervous system functions. ${ }^{38}$ Androgens as glucocorticoids could have mild suppressive effects on the HPA axis and on the immune and inflammatory reaction, whereas oestrogens may have the opposite effect, possibly enhancing the sexual dimorphism of the stress, immune and inflammatory reactions. ${ }^{39}$

In a meta-analysis of 45 studies, Otte et al showed that the effect of age on cortisol response could be threefold stronger in women than men. ${ }^{40}$ Regarding anxiety disorder, we have previously shown, in a subsample of the ESPRIT cohort, that participants, especially women, with GAD or phobia had higher diurnal cortisol levels and lowered recuperation capacity in response to an environmental stress compared with participants without anxiety. ${ }^{41}$ Anxiety has also been reported to be associated with inflammation and coagulation markers ${ }^{42}$ and low-grade systemic inflammation is related to mortality in healthy elderly men and women. ${ }^{43}$ Other markers of chronic stress and HPA axis hyperactivity, including insulin resistance, endothelial reactivity and altered autonomic function, have been evoked as the mechanisms linking anxiety in elderly people and cardiovascular disease. ${ }^{44}$ This hypothesis is also compatible with our observation of a possible increased risk of cardiovascular mortality associated with anxiety.

\section{Limitations and strengths}

Bias may have been introduced by the non-random exclusion of participants with missing data at baseline who were less likely to have anxiety disorder and symptoms. Relatively small sample sizes when analysing subtypes of anxiety disorder or causes of death may also yield unstable estimates. In spite of extensive adjustments for a large number of potential confounding factors, the possibility remains with observational data that unmeasured confounders such as individual behaviour or social environment may partly explain the associations. Also, comorbidity was assessed indirectly by standardised questions and medication use without clinical examination.

Our prospective study based on a large community sample has several strengths. It permitted stratification by gender and adjustment for a wide range of possible confounders including depression, which was found to be a key confounding factor. We were able to obtain differential diagnosis of specific anxiety disorders using a standardised psychiatric examination based on DSM-IV criteria. The statistical power for detecting a significant effect in men and in women is comparable as a result of a similar 
number of death events. The causes of death were established by an independent committee. The survival analysis, using a statistical model with delayed entry, was best able to take into account age effects, which is critical in elderly samples.

\section{Implications}

Our study has demonstrated a significant association between anxiety disorder and all-cause mortality in women only, which may be explained by endocrinal factors. This gender-specific effect along with the observed variability in findings according to whether anxiety is defined as dimensional symptomatology or a categorical psychopathology may explain many of the inconsistencies in previous observations. These factors should be taken into account in future research.

Isabelle Carrière, PhD, Inserm U1061 and University of Montpellier I, Montpellier, France; Joanne Ryan, PhD, Inserm U1061 and University of Montpellier I, Montpellier, France, and Murdoch Children's Research Institute, Royal Children's Hospital, Parkville, Victoria, Australia; Joanna Norton, PhD, Jacqueline Scali, MSC, Inserm U1061 and University of Montpellier I, Montpellier, France; Robert Stewart, PhD, Institute of Psychiatry, King's College London, UK; Karen Ritchie, PhD, Inserm U1061 and University of Montpellier I, Montpellier, France, and Faculty of Medicine, Imperial College, St Mary's Hospital, London, UK; Marie Laure Ancelin, PhD, Inserm U1061 and University of Montpellier I, Montpellier, France

Correspondence: Isabelle Carrière, Inserm U1061, Neuropsychiatry: Epidemiological and Clinical Research, Hôpital La Colombière, 39 Avenue Charles Flahault, BP 34493, 34093 Montpellier cedex 05, France. Email:

isabelle.carriere@inserm.fr

First received 6 Dec 2012, final revision 3 Apr 2013, accepted 8 May 2013

\section{Funding}

The $3 \mathrm{C}$ Study is conducted under a partnership agreement between the Institut Nationa de la Santé et de la Recherche Médicale (Inserm), Victor-Segalen Bordeaux2 University, and sanofi-aventis. The 3C-Study was also supported by the Caisse Nationale Maladie des Travailleurs Salariés, Direction Générale de la Santé, MGEN, the Institut de la Longévité des Travailleurs Salariés, Direction Générale de la Santé, MGEN, the Institut de la Longévité, of Aquitaine, Bourgogne and Languedoc-Roussillon, the Fondation de France, the Ministry of Research-Inserm Programme 'Cohorts and collection of biological material', the 'ANR Agence Nationale de la Recherche - The French National Research Agency' under the 'Programme National de Recherche en Alimentation et nutrition humaine', project 'COGINUT ANR-06-PNRA-005', the 'Programme Longévité et vieillissement', project 07 LVIE-004 and 07-LVIE 003 01, the Institut de Recherche en Santé Publique (IReSP), Paris, France and Novartis. R.S. is part-funded by the National Institute for Health Research (NIHR) Biomedical Research Centre at South London and Maudsley NHS Foundation Trust and King's College London.

\section{References}

1 Cuijpers $P$, Schoevers RA. Increased mortality in depressive disorders: a review. Curr Psychiatry Rep 2004; 6: 430-7.

2 Schulz R, Drayer RA, Rollman BL. Depression as a risk factor for non-suicide mortality in the elderly. Biol Psychiatry 2002; 52: 205-25.

3 Dewey ME, Chen CM. Neurosis and mortality in persons aged 65 and over living in the community: a systematic review of the literature. Int J Geriatr Psychiatry 2004; 19: 554-7.

4 Holwerda TJ, Schoevers RA, Dekker J, Deeg DJ, Jonker C, Beekman AT. The relationship between generalized anxiety disorder, depression and mortality in old age. Int J Geriatr Psychiatry 2007; 22: 241-9.

5 Markkula N, Härkänen T, Perälä J, Partti K, Peña S, Koskinen S, et al. Mortality in people with depressive, anxiety and alcohol use disorders in Finland. Br J Psychiatry 2012; 200: 143-9.

6 Eaker ED, Sullivan LM, Kelly-Hayes M, D'Agostino Sr RB, Benjamin EJ. Tension and anxiety and the prediction of the 10-year incidence of coronary heart disease, atrial fibrillation, and total mortality: the Framingham Offspring Study. Psychosom Med 2005; 67: 692-6.

7 Lavretsky H, Mistry R, Bastani R, Gould R, Gokhman I, Huang D, et al. Symptoms of depression and anxiety predict mortality in elderly veterans enrolled in the UPBEAT program. Int J Geriatr Psychiatry 2003; 18: 183-4.

8 van Hout HPJ, Beekman ATF, de Beurs E, Comijs $\mathrm{H}$, van Marwijk $\mathrm{H}$, de Haan $\mathrm{M}$, et al. Anxiety and the risk of death in older men and women. Br J Psychiatry 2004; 185: 399-404.
9 Denollet J, Maas K, Knottnerus A, Keyzer JJ, Pop VJ. Anxiety predicted premature all-cause and cardiovascular death in a 10-year follow-up of middle-aged women. J Clin Epidemiol 2009; 62: 452-6.

10 Laan W, Termorshuizen F, Smeets HM, Boks MP, de Wit NJ, Geerlings MI. A comorbid anxiety disorder does not result in an excess risk of death among patients with a depressive disorder. J Affect Disord 2011; 135: 284-91.

11 Ostir GV, Goodwin JS. High anxiety is associated with an increased risk of death in an older tri-ethnic population. J Clin Epidemiol 2006; 59 : $534-40$.

12 Phillips AC, Batty GD, Gale CR, Deary IJ, Osborn D, Macintyre K, et al. Generalized anxiety disorder, major depressive disorder, and their comorbidity as predictors of all-cause and cardiovascular mortality: the Vietnam experience study. Psychosom Med 2009; 71: 395-403.

13 Batterham PJ, Christensen $\mathrm{H}$, Mackinnon AJ. Mental health symptoms associated with morbidity, not mortality, in an elderly community sample. Soc Psychiatry Psychiatr Epidemiol 2012; 47: 79-85.

14 Mykletun A, Bjerkeset O, Dewey M, Prince M, Overland S, Stewart R. Anxiety, depression, and cause-specific mortality: the HUNT study. Psychosom Med 2007; 69: 323-31.

15 Ritchie K, Norton J, Mann A, Carrière I, Ancelin ML. Late-onset agoraphobia: general population incidence and evidence for a clinical subtype. Am J Psychiatry 2013; 170: 790-8.

16 Thiebaut AC, Benichou J. Choice of time-scale in Cox's model analysis of epidemiologic cohort data: a simulation study. Stat Med 2004; 23: 3803-20.

17 Ritchie K, Artero S, Beluche I, Ancelin ML, Mann A, Dupuy AM, et al. Prevalence of DSM-IV psychiatric disorder in the French elderly population Br J Psychiatry 2004; 184: 147-52.

18 World Health Organization. The ICD-10 Classification of Mental and Behavioural Disorders: Clinical Descriptions and Diagnostic Guidelines. WHO, 1992.

19 Sheehan DV, Lecrubier $\mathrm{Y}$, Sheehan $\mathrm{KH}$, Amorim $\mathrm{P}$, Janavs J, Weiller $\mathrm{E}$, et al. The Mini-International Neuropsychiatric Interview (M.I.N.I.): the development and validation of a structured diagnostic psychiatric interview for DSM-IV and ICD-10. J Clin Psychiatry 1998; 59 (suppl 20): 22-33; quiz 34-57.

20 Lecrubier $Y$, Sheehan D, Weiller E, Amorim P, Bonara I, Sheehan K, et al. The Mini International Neuropsychiatric Interview (MINI), a short diagnostic interview: reliability and validity according to the CIDI. Eur Psychiatry 1997; 12: $232-41$

21 American Psychiatric Association. Diagnostic and Statistical Manual of Mental Disorders (4th edn) (DSM-IV). APA, 1994.

22 Spielberger C. Manual for the Sate-Trait Anxiety Inventory (Form Y). Consulting Psychologists Press, 1983

23 Radloff L. The CES-D Scale: a self-report depression scale for research in the general population. Appl Psychol Meas 1977; 1: 385-401.

24 Ryan J, Carriere I, Ritchie K, Stewart R, Toulemonde G, Dartigues JF, et al. Late-life depression and mortality: influence of gender and antidepressant use. $\mathrm{Br} J$ Psychiatry 2008; 192: 12-8.

25 Folstein MF, Folstein SE, McHugh PR. "Mini-mental state". A practical method for grading the cognitive state of patients for the clinician. I Psychiatr Res 1975; 12: 189-98.

26 Ancelin ML, Carriere I, Boulenger JP, Malafosse A, Stewart R, Cristol JP, et al. Gender and genotype modulation of the association between lipid levels and depressive symptomatology in community-dwelling elderly (the ESPRIT study). Biol Psychiatry 2010; 68: 125-32.

27 Rosow I, Breslau N. A Guttman health scale for the aged. J Gerontol 1966; 21: $556-9$.

28 Lawton MP, Brody EM. Assessment of older people: self-maintaining and instrumental activities of daily living. Gerontologist 1969; 9: 179-86.

29 Mykletun A, Bjerkeset O, Øverland S, Prince M, Dewey M, Stewart R. Levels of anxiety and depression as predictors of mortality: the HUNT study. Br J Psychiatry 2009; 195: 118-25.

30 Roest AM, Martens EJ, Denollet J, de Jonge P. Prognostic association of anxiety post myocardial infarction with mortality and new cardiac events: a meta-analysis. Psychosom Med 2010; 72: 563-9.

31 Meyer T, Buss U, Herrmann-Lingen C. Role of cardiac disease severity in the predictive value of anxiety for all-cause mortality. Psychosom Med 2010; 72: 9-15.

32 Watkins LL, Blumenthal JA, Babyak MA, Davidson JR, McCants Jr CB, $\mathrm{O}$ 'Connor $\mathrm{C}$, et al. Phobic anxiety and increased risk of mortality in coronary heart disease. Psychosom Med 2010; 72: 664-71.

33 Flint AJ. Anxiety and its disorders in late life: moving the field forward. Am J Geriatr Psychiatry 2005; 13: 3-6. 
34 Wolitzky-Taylor KB, Castriotta N, Lenze EJ, Stanley MA, Craske MG. Anxiety disorders in older adults: a comprehensive review. Depress Anxiety 2010; 27 190-211.

35 Gill TM, Gahbauer EA, Han L, Allore HG. Trajectories of disability in the last year of life. N Engl J Med 2010; 362: 1173-80.

36 Ryan J, Scali J, Carriere I, Scarabin PY, Ritchie K, Ancelin ML. Estrogen receptor gene variants are associated with anxiety disorders in older women. Psychoneuroendocrinology 2011; 36: 1582-6.

37 Ryan J, Canonico M, Carcaillon L, Carriere I, Scali J, Dartigues JF, et al. Hormone treatment, estrogen receptor polymorphisms and mortality: a prospective cohort study. PLOS One 2012; 7: e34112.

38 Chrousos GP. Stress and disorders of the stress system. Nat Rev Endocrinol 2009; 5: 374-81.

39 Chrousos GP. Stress and sex versus immunity and inflammation. Sci Signal 2010; 3: pe36.
40 Otte C, Hart S, Neylan TC, Marmar CR, Yaffe K, Mohr DC. A meta-analysis of cortisol response to challenge in human aging: importance of gender. Psychoneuroendocrinology 2005; 30: 80-91.

41 Chaudieu I, Beluche I, Norton J, Boulenger JP, Ritchie K, Ancelin ML. Abnormal reactions to environmental stress in elderly persons with anxiety disorders: evidence from a population study of diurnal cortisol changes. J Affect Disord 2008; 106: 307-13.

42 Pitsavos C, Panagiotakos DB, Papageorgiou C, Tsetsekou E, Soldatos C, Stefanadis C. Anxiety in relation to inflammation and coagulation markers, among healthy adults: the ATTICA study. Atherosclerosis 2006; 185: 320-6

43 Carriere I, Dupuy AM, Lacroux A, Cristol JP, Delcourt C. Biomarkers of inflammation and malnutrition associated with early death in healthy elderly people. J Am Geriatr Soc 2008; 56: 840-6.

44 Lenze EJ, Wetherell JL. A lifespan view of anxiety disorders. Dialogues Clin Neurosci 2011; 13: 381-99. 